29. Appel-Dingemanse S, Lemarechal MO, Kumle A, Hubert M, Legangneux E. Integrated modelling of the clinical pharmacokinetics of SDZ HTF 919, a novel selec-tive 5-HT4 receptor agonist, following oral and intravenous administration. Br J Clin Pharmacol 1999;47: 483-91.

\title{
IMAGE
}

\section{Blind pouch syndrome following enterocolic anastomosis - multidetector computed tomographic findings}

A 60-year-old man with complaints of diarrhea and a previous history of colonic carcinoma which was operated several years back underwent a multidetector computed tomographic (MDCT) scan of the abdomen which revealed a subtotal colectomy status with preservation of the sigmoid colon and the rectum. A side-to-side anastomosis between the ileum and the sigmoid colon was noted (Fig. 1). The blind end of the anastomosed ileal loop was dilated and contained mottled contents suggestive of retained fecal matter. There was surrounding minimal mesenteric fat stranding (Fig. 2) and mesenteric lymphadenopathy. The CT features were suggestive of post-surgical blind pouch of the small bowel.

Blind pouch syndrome is a well-recognized complication of small bowel anastomosis. Abnormal peristalsis results in filling of the pouch, with stagnation, bacterial overgrowth and subsequent diarrhea. ${ }^{1}$ These are most commonly seen after side-to-side anastomosis. ${ }^{1}$ It is important to identify them as complications such as small bowel obstruction. enteroliths, ulceration, bleeding and anemia are known to occur. ${ }^{1}$ Distinguishing a diverticulum from a blind pouch may be difficult on CT. However identification of adjacent surgical clips helps to confirm the diagnosis. ${ }^{1}$ The maximum sac diameter

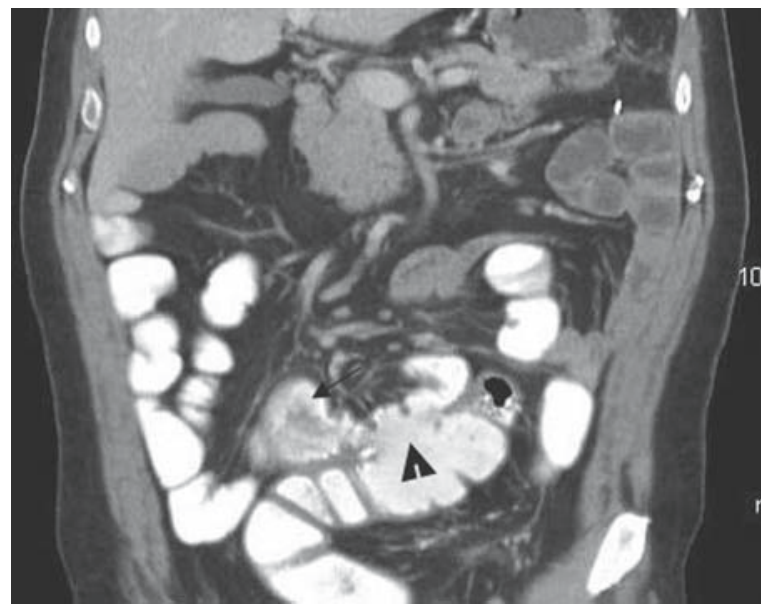

Fig. 1 Coronal reformatted MDCT study of the abdomen reveals post enterocolic anastomosis (black arrow head). The blind end of the anastomosed ileal loop is dilated containing fecal residue (black arrow) in proven cases of blind pouch syndrome in a previous study ${ }^{1}$ ranged between $3.7 \mathrm{~cm}$ to $11 \mathrm{~cm}$. In our case the maximum sac diameter was $4.4 \mathrm{~cm}$. The mesenteric fat stranding and the enlarged mesenteric lymphnodes support the diagnosis of bacterial infection within the pouch ${ }^{1}$ and are not seen in an otherwise uncomplicated case of side-to-side anastomosis.

Nabil Sherif Mahmood Hadihally Byregowda Suresh · Vinod Hegde Department of Radiodiagnosis and Imaging, Father Muller Medical College, Mangalore 575 002, Karnataka, India

N. S. Mahmood $(\square)$ e-mail: nabilsherifmahmood@rediffmail.com

\section{Reference}

1. Sandrasegaran K, Maglinte DD, Rajesh A, et al. CT findings for postsurgical blind pouch of small bowel. AJR Am J Roentgenol 2006;186:110-3.

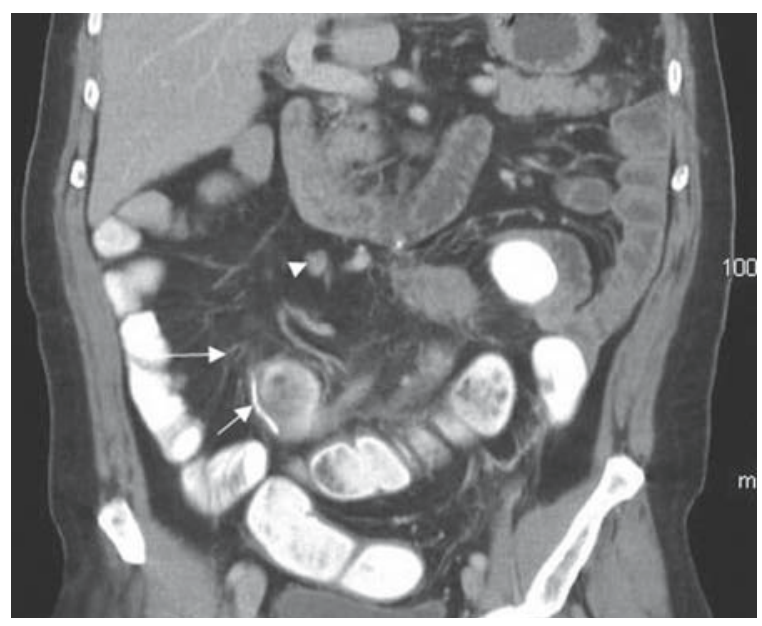

Fig. 2 Coronal reformatted MDCT study reveals surgical suture at the distal end of the blind pouch (short white arrow). There is associated mesenteric fat stranding (long white arrow) and lymphadenopathy (white arrowhead) 